"C2020 IEEE. Personal use of this material is permitted. Permission from IEEE must be obtained for all other uses, in any current or future media, including reprinting/republishing this material for advertising or promotional purposes, creating new collective works, for resale or redistribution to servers or lists, or reuse of any copyrighted component of this work in other works." 


\title{
ICFHR 2020 Competition on Short answer ASsessment and Thai student SIGnature and Name COMponents Recognition and Verification (SASIGCOM 2020)
}

\author{
Abhijit Das $^{\mathrm{a}}$, Hemmaphan Suwanwiwat ${ }^{\mathrm{b}}$, Umapada Pal ${ }^{\mathrm{a}}$, and Michael Blumenstein ${ }^{\mathrm{c}}$ \\ ${ }^{a}$ Computer Vision and Pattern Recognition Unit, Indian Statistical Institute, Kolkata, India, abhijitdas2048@gmail.com, \\ umapada@isical.ac.in \\ bInformation Technology Academy, James Cook University, Cairns, Australia, art.suwanwiwat@jcu.edu.au \\ ${ }^{\mathrm{c} S c h o o l ~ o f ~ S o f t w a r e, ~ U n i v e r s i t y ~ o f ~ T e c h n o l o g y ~ S y d n e y, ~ A u s t r a l i a, ~ m i c h a e l . b l u m e n s t e i n @ u t s . e d u . a u ~}$
}

\begin{abstract}
This paper describes the results of the competition on Short answer ASsessment and Thai student SIGnature and NameCOMponents Recognition and Verification (SASIGCOM 2020) in conjunction with the 17th International Conference on Frontiers in Handwriting Recognition (ICFHR 2020). The competition was aimed to automate the evaluation process short answer-based examination and record the development and gain attention to such system. The proposed competition contains three elements which are short answer assessment (recognition and marking the answers to short-answer questions derived from examination papers), student name components (first and last names) and signature verification and recognition. Signatures and name components data were collected from 100 volunteers. For the Thai signature dataset, there are 30 genuine signatures, 12 skilled and 12 simple forgeries for each writer. With Thai name components dataset, there are $\mathbf{3 0}$ genuine and 12 skilfully forged name components for each writer. There are 104 exam papers in the short answer assessment dataset, 52 of which were written with cursive handwriting; the rest of 52 papers were written with printed handwriting. The exam papers contain ten questions, and the answers to the questions were designed to be a few words per question. Three teams from distinguished labs submitted their systems. For short answer assessment, word spotting task was also performed. This paper analysed the results produced by their algorithms using a performance measure and define a way forward for this subject of research. Both the datasets, along with some of the accompanying ground truth/baseline mask will be made freely available for research purposes via the TC10/TC11.
\end{abstract}

Keywords- handwriting recognition, Thai signature and name component recognition and verification, short answer assessment

\section{INTRODUCTION}

The competition's aim is to automate the evaluation process of short answer-based examination. Such automation will benefit education from, not only the significant time reduction when marking short answer examination papers, but also in being able to identify and verify that a student's signing signature on an exam paper is genuine and is their own. Typically, an answering script from short answer typeexamination consists of three handwritten components which are student name, signature, and short (few-word) answers. Hence, tasks for this competition are short answer assessment (recognition or word-spotting of the short-answer questions derived from examination papers), student name components (first and last names) and signature recognition and verification. There is still a limited amount of research regarding off-line automatic marking systems found in the literature [1-3], though till date paper-based examinations are still practically used world-wide. This is because not every education institute in the world can afford enough number of computers for students per class because of potentially high costs. Additionally, in larger classes, marking examination papers can be a difficult, prolonged and tiring task. As a result, a successful off-line automatic assessment system could be utilised to assist in marking so that the time and efforts used for marking could be reduced.

To the best of the organisers' knowledge, there has never been a competition on off-line short answer assessment before. As a result, this competition may be able to find efficient or new techniques in recognising or word spotting such handwritten short answers. For this competition, a short answer words dataset was proposed.

Biometric traits for identity verification are including but not limited to vein $[4,10]$, signature and name components [5], fingerprint [6], face [7], iris [8] and voice [5]. Forensic Handwriting Experts (FHE) can perform manual verification; however, it could be a very long and tedious process. Automatic Signature Verification systems (ASVs) can be employed to tackle the above problems of manual signature verification [9].

Signatures are used daily by people in Thailand as proof of their identities. Signatures signed by students who are 16 years of age and above are also being used in schools and universities. Students' signatures together with their name components, being first and last names can be found in exam answer sheets. This competition on Thai student signature and name component datasets aims to find novel or efficient techniques in identifying and verifying student name components and student signatures. The competition could be considered challenging as students' handwriting are less consistent compared to adults' ones. As described above, there are three (3) datasets for the participants to choose and employ with their proposed techniques. The datasets are the proposed short answer word dataset, a Thai student name components dataset and a Thai student signature dataset. The tasks which the participants could participate in this competition are short answer word spotting/recognition, signature recognition and verification, and student name components recognition and verification.

The discussed areas of research above can be an emerging point of interest to give a paradigm shift to the education system. Therefore, to prove their feasibility and usefulness, scientific attention and efforts are required. This competition also aims to establish a standard benchmark and promote automatic short answer assessment, Thai student signature and name components recognition and verification. To achieve the above aims, the SASIGCOM 2020 was organised in conjunction with the $17^{\text {th }}$ International Conference on Frontiers in Handwriting Recognition (ICFHR 2020) as it is the biggest gathering of the handwriting research community.

The rest of this paper is organised as follows. In Section II, the datasets for the competition and the performance 
evaluation technique adopted to evaluate and rank the participants' algorithms are described. In Section III, various algorithms from the participants are described in detail, in Section IV, the results achieved from the submitted algorithms and their detailed analysis is summarised. Finally, in Section $\mathrm{V}$, the overall conclusions are drawn, and future work is discussed.

\section{SASIGCOM 2020 COMPETITION DETAILS}

The competition details, including competition participants, competition datasets, and tasks description, are summarised in this section.

\section{A. The competition participants}

The competition was promoted through a website** and by emails. Six (6) applications from distinguished laboratories of academia and industry around the world were received. Among them, four (4) of which submitted their algorithms; the details of the participating teams and their affiliations are displayed in TABLE I.

TABLE I. DESCRIPTIONS OF PARTICIPATING TEAMS AND THEIR AFFILIATIONSD

\begin{tabular}{|c|c|}
\hline Team & Name (Institution) \\
\hline SCUT & $\begin{array}{l}\text { Songxuan Lai, Yecheng Zhu, Jiajia Jiang, Lianwen Jin (South } \\
\text { China University of Technology) }\end{array}$ \\
\hline $\begin{array}{l}\text { LMU- } \\
\text { UAM- } \\
\text { ULPGC }\end{array}$ & $\begin{array}{l}\text { Walid Bouamra }{ }^{1} \text {, Moises Diaz }{ }^{2} \text {, Miguel A. Ferrer }{ }^{3} \text {, Brahim } \\
\text { Nini }^{1} \text { : }{ }^{1} \text { Larbi Ben M'hidi University, Algeria, }{ }^{2} \text { Universidad } \\
\text { del Atlantico Medio, Spain, }{ }^{3} \text { Universidad de Las Palmas de } \\
\text { Gran Canaria, Spain. }\end{array}$ \\
\hline $\begin{array}{l}\text { UoB- } \\
\text { UoT }\end{array}$ & $\begin{array}{l}\text { Ismail Hadjadj1,2, Abdeljalil } \text { Gattal }^{2} \text {, Chawki Djeddi }{ }^{2} \text {, } \\
\text { Mouloud Ayad }{ }^{1} \text {, Faycel Abbas }{ }^{1,2}:{ }^{1} \text { University Of Bouira, } \\
\text { Algeria, }{ }^{2} \text { University Of Tebessa, Algeria }\end{array}$ \\
\hline IF & Ltd,, Bangal \\
\hline
\end{tabular}

\section{B. Competition Datasets}

There are three (3) datasets used in this competition being, Thai student signature dataset, Thai student name components dataset, and short answer word dataset. For name component and student signatures, each volunteer wrote their signature/name 30 times, using the motion time interval technique in the given space on white paper. They signed their signatures, as they usually did, ten signatures at a time, and then pause. After a short moment of pause, they repeated the process two more times. The time interval was for a few minutes. It was used so that the signers would not feel too tired. As a result, natural signatures (as much as possible), without much deformation, could be obtained.

The short answer spotting/recognition task utilised the proposed short answer dataset. The aim of this task was for the participants to be able to spot the correct answer words. There are two (2) types of handwriting in the dataset which are printed and cursive handwriting. The exam papers contained ten (10) questions; the answer to each question was designed to be a few words (1-3 words) per question and was straightforward. An example of the exam questions is "What does IT stand for?", The correct answer can only be "Information Technology", although the writers may write words using different cases, such as "information technology" and "Information technology". The nature of these exam papers makes it suitable to be used for short answer question assessment systems [19]. Figure 1 displays some examples of handwritten short answers.

\begin{tabular}{|c|c|c|c|}
\hline Word & Handwritten Word Sample 1 & Handwritten Word Sample 2 & Handwritten Word Sample 3 \\
\hline Information & Infonmation & informmotion & INFORMATION \\
\hline Input & Shput & INPUT & InRAt \\
\hline Programming & Prongrwonsing & Progrannming & PROGRAMMING \\
\hline Database & Dakabase & dutabose & Qutabace \\
\hline $\begin{array}{l}\text { Misspelt } \\
\text { words }\end{array}$ & Datababase & Lazer & Languge \\
\hline
\end{tabular}

Fig. 1. Examples of short answers.

For Thai student signatures dataset [12], both Genuine Signature (GS) and forged Thai student signatures were obtained from 100 students. As a result, the whole dataset contains 3,000 (100 signer's $\times 30$ times $)$ genuine signatures in this dataset. For each of the genuine signatures, 12 skilfully and 12 simple forged signatures were produced; therefore, there are 24 forged signatures per each genuine signer. In total, there are 1,200 (100 signer's $\times 12$ times) skilfully forged, and $1,200$ (100 signers $\times 12$ times $)$ simple forged signatures. Altogether, there are 5,400 signatures in this dataset.

Skilled Forged Signers (SFS) were asked to learn to forge genuine signatures of the other genuine signers. By learning, they tried to copy the genuine signatures (practised signing the genuine signatures) until they felt confident in forging the genuine signatures. Once they were confident, they provided skilled forged signatures to the collector.

Simple Forgeries Signature (SiFS) are in a set of 12 signatures per user with similar vocal outcomes as the original. The forger only knew how the name sounded and had to decide how to sign the given name as the signature can be signed in several ways, e.g. signed in Thai, English, Mixed, or signed by just simply writing down the name (so more like writing rather than signing). The occasions which the signature from a simple forger looks very close to the genuine signature are rare.

It was found that 31 volunteers signed their signatures in English script, whereas the other 64 signed their signatures in Thai and five (5) signers used both scripts to sign a single signature. All samples were scanned at $300 \mathrm{dpi}$ and are binarised. Student signatures, characteristics and their examples can be seen in Figure 2.

For Thai name components (TNC) dataset [13], there are 6,000 (100 students $\times 2$ name components $\times 30$ times) genuine name components collected. For each of the genuine name components, 12 skilfully forged name components were produced. In total, there are 2,400 (100 students $\times 2$ name components $\times 12$ times) skilfully forged. Altogether, there are 8,400 name components in this dataset. The Thai name components [13], both genuine and forged, were obtained from 100 students, whose ages were between 12 and 16 years old.

Each student was asked to write their name (first and last name) 30 times, using the motion time interval technique (described under Thai student signatures Sub-section), on white paper in the given space. All samples were scanned at $300 \mathrm{dpi}$ and are binarised. The Thai name component dataset was collected from different groups of volunteers than those who signed the signatures in the signature dataset. Examples of genuine and skilfully forged Thai name components can be seen in Figure 3.

**http://sasigcomp2020.chefitup.club/art.suwanwiwat/ 


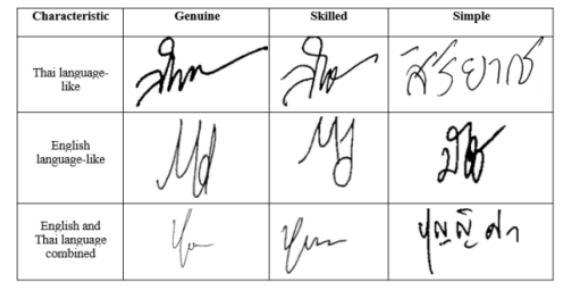

Fig. 2. Examples of Signature Characteristics.

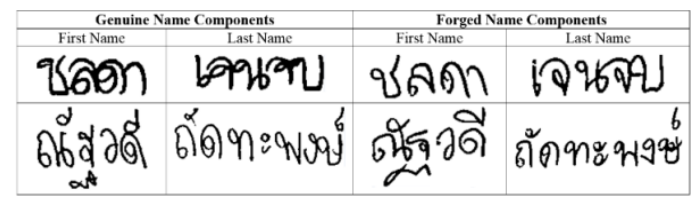

Fig. 3. Examples of genuine and skilfully forged Thai name components

Short answer dataset [11]:_There are 104 exam papers in this dataset, 52 of which were written using cursive handwriting; the rest of 52 papers were written with printed handwriting. This dataset was used for the word spotting task, where known words (corresponding to the correct answers) should be spotted then marked. Two sub-datasets of each handwritten type were offered to the participants to develop their algorithms for the task. Ten (10) exam papers from ten (10) writers were used for training.

For each task, the competitors employed a corresponding training subset(s) for training. The numbers of samples for training, which were taken from their whole dataset, are described in Table II. The testing samples from each dataset were used for testing the competitors' submissions. Technical details about these datasets are summarised in Table II.

TABLE II. DETAILS OF EACH TRAINING DATASETS (TAKEN FROM THE WHOLE DATASETS)

\begin{tabular}{|c|c|c|c|c|}
\hline Dataset & $\begin{array}{c}\text { No. of } \\
\text { users }\end{array}$ & $\begin{array}{c}\text { GS } \\
\text { sample }\end{array}$ & $\begin{array}{c}\text { SFS } \\
\text { Sample }\end{array}$ & $\begin{array}{c}\text { SiFS } \\
\text { sample }\end{array}$ \\
\hline Signature First subset & 30 & 30 & 12 & 12 \\
\hline Signature second subset & 100 & 5 & 5 & 5 \\
\hline NC First subset & 30 & 30 & 12 & N/A \\
\hline NC second subset & 100 & 5 & 5 & N/A \\
\cline { 1 - 2 } Short answer First subset & 10 & & & NA \\
\cline { 1 - 2 } Short answer Second subset & 10 & \multicolumn{5}{|r}{} \\
\cline { 1 - 3 }
\end{tabular}

\section{A. Tasks Description}

There were six (6) tasks in this competition which the participants could participate in single or multiple tasks using the three types of datasets provided; these available tasks were:

\section{1) Short Answer Assessment}

- Recognise the answers of the word spotted.

- Spotting the appropriate answer in the exam paper.

2) Thai Student Name Components Recognition and Verification

- Identify students by employing Thai student handwritten name components (first and last names).
- Verify students by employing Thai student handwritten name components (first and last names).

3) Thai Student Signature (signed by the 16 and aboveyears of age) Recognition and Verification.

- Identify students by employing Thai student signatures.

- Verify students by employing Thai student signatures

The participants were asked to submit training and testing scripts/algorithms for the recognition tasks. The training script can read training images from a folder (names/signatures) and generate the model, and the testing script can take the test image and generate the predicted level/class the test image belongs to. For the verification task, the participants were asked to submit a script that can read the training images and a test image and generate its verification score. For the recognition task, the Recognition Accuracy (RA) was considered for the performance measure. For the verification task, the Equal Error Rate (EER) was used as the performance measure. The respective computation protocol is described in the experimental protocol sub-section.

For word spotting task, the algorithms submitted by the participants were evaluated for the precision measure, recall and F-measure (the F-measure was considered as the primary measure for ranking the submitted models). The manually annotated ground truth (i.e., the word region in the images) was used as the basis for scoring.

\section{BRIEF DESCRIPTION OF THE SUBMITTED ALGORITHMS}

The word spotting and signature and name component recognition and verification algorithms submitted by the four participated teams are described in this section.

A. Name and signature recognition amd verification algorithm: Submitted by Team SCUT

SCUT-CNN: Similar to [14], the CNN network in [15] with fractional max pooling [16] and image moments-based size normalisation [17] was to train and classified with kernel ridge regression. To train the $\mathrm{CNN}$, they employed several publicly available databases, including MCYT-75, CEDAR, BiosecurID-SONOF-DB and SigComp11, and a private Chinese signature database, leading to 13,987 training signatures in total.

B. Name, signature and answer recognition and verification algorithm: Submitted by Team LMU-UAM-ULPGC

This team submitted four algorithms they are as follows:

1. Run Length (RL) 400 Algorithm (RL400) [18]: A run is equivalent to a segment which is a sequence of white or black pixels. The run-length matrix is defined as a matrix $\mathrm{P}$, where the value at position $(i, j)$ in the matrix represents the number of pixels runs of colour $i$ and length $j$ in a given direction. The size of the matrix is $\mathrm{M} \times \mathrm{K}$, where $\mathrm{M}$ represents the number of unique colours (intensities) in the image while $\mathrm{K}$ is the maximum possible length of a run-in a given direction. In their study, they considered the horizontal, vertical, leftdiagonal and right-diagonal run-lengths on black and white pixels of the binarised images of signatures. The final Run Length vector is composed of 400 values.

2. Extended Run Length Algorithms (ERL): This is an extension of standard run-length features. The standard run- 
length vector is about 400 values for the four directions. While the total extended RL vector is composed of 3,200 values (which is the addition of 400 black pixels and 400 white pixels of the direction $0^{\circ}, 45^{\circ}, 90^{\circ}$, and $135^{\circ}(800$ pixels $\times 4$ directions $=3,200$ values $)$ ). The final Run Length vector is composed of 3,200 values.

3. Multidirectional Run Length Algorithm (MDRL): This is based on Run-Length distributions adding four directions to the four standard directions. In total, they get eight composite directions; each one is composed of a combination of three neighbouring angles.

4. Spiral Run Length Algorithm (SRL): This is based on the standard run-length features using only two directions which are horizontal and vertical. It is described by a uniform displacement on a rotating line itself uniform until reaching a final point; the spiral run-length algorithm traverses the entire image in a spiral curve starting from the first pixel at the upper left corner of the image, then moves away more and more towards a last central point, as it turns around. The same standard Run-length algorithm is applied to the resulting vector on spiral function. The final Spiral RL vector size is about 800 (400 for black pixels +400 for white pixels).

\section{Signature verification algorithm: Submitted by Team UoB-UoT}

This team submitted four algorithms they are as follows:

1. BIF: This system is based on oriented Basic Image Features (oBIFs) an extension of the Basic Image Features (BIFs) that involves combining the local orientation with the local symmetry information.

2. LTP: This system is based on Local Ternary Pattern (LTP). The key idea of the LTP descriptor is to extend the two (2) valued $(0 ; 1)$ Local Binary Pattern (LBP) codes to three values $(-1 ; 0 ; 1)$ based on a threshold. The upper and lower threshold values are defined around the basic threshold. Neighbouring pixels (with reference to the central pixel) taking values between these thresholds are assigned with 0 ; value of 1 is assigned to the pixels with value greater than the upper threshold; and value of -1 otherwise.

3. LTP+oBIFs [19]: In this system, images are characterised using two textural descriptors, the Local Ternary Patterns (LTP) and the oriented basic image features (oBIFs).

4. RL: The proposed system is derived from the work presented in [20]. The multi-scale run-length features are used to describe the binary images of the signature, where black pixels presented the ink trace, and the background is presented with the white pixels.

\section{Short answer spotting algorithm: Submitted by Team IF}

Orientation encoding of square kernels: An image is divided into $9 \times 9$ tiles, where each tile is of a size of $15 \times 15$ pixels. One feature is computed and stored for each square. The tagged bounding box information is used to set whether the pixel is a part of handwritten word or printed word along with the pixel position and the features. A database of 86 attributes (81 features, 1 magnitude of features, 2 pixel positions, 2 class attribute and information) is created from the training set images. The features are extracted from the testing image and compared with the database using crosscorrelation. The class of the highest correlation value is assigned to the test pixel. A stride length of 5 pixels is used to reduce the computational load. Each image takes around 90 minutes to complete the pixel classification task. The assigned class labels of test pixels are dilated to cover the pixels which were not a part of the classification. The dilated image is filtered to retain components with size more than 50 $\times 50$ pixels. The morphological operation of closing and opening is performed to cover the holes within the image components. The components with xmin of the bounding box that are less than 500 are removed to avoid handwritten numbers in the documents. The bounding box of all the remaining components is grouped using horizontal overlap between the components. The bounding box of the components is used with the original image to find the actual handwritten word boundary. A one-pixel gap is provided around the spotted handwritten words to refine the detected bounding boxes.

\section{RESULTS AND DISCUSSION}

This section includes the experiment protocol, a report on results achieved, an evaluation of the submitted algorithm on the tasks each team had chosen, a report on the analysis and discussion on the way forward on this topic of research to resolve the identified research challenges.

\section{A. Experimental protocol}

For signature and name component verification tasks, the verifiers were trained with the first five (5) genuine samples of each writer/signer of the corresponding datasets for repeatability of the experiments and in $[12,13]$. The rest of the genuine samples were used for testing the false rejection rate. The false acceptance rate for the random forgeries was obtained from using the genuine testing samples from all the remaining writers/signers. The false acceptance rate for the skilled and simple forgery experiments was attained by using all the forgery samples of each writer/signer. With the recognition tasks, the first 5 genuine samples from each user were used for training and the rest of genuine samples were used for testing.

\section{B. Performance measures}

As per the competition protocol, for the signature recognition tasks, the algorithms were ranked by their performance accuracy rates. For the name component recognition, the best Recognition Accuracy (RA) rate, among last name ( $\mathrm{LN})$ or first name $(\mathrm{FN})$ or first and last name combined, was considered for the ranking. The average EER of random, skilled, and simple forgeries of each verifier was considered for the signature verification task ranking.

Similar to name component recognition task, for the name component verification task, the best average EER of random (RFS) and skilled forgery experiment of each algorithm, considering first name or last name, or first and last name combined, was considered for algorithms ranking.

For word spotting, the precision measure, recall and Fmeasures were used for evaluation. It can be noted that the Fmeasure was considered the primary measures for ranking the submitted models. For answer recognition task RA was employed.

\section{Results of the submitted algorithms}

Results attained from the signature verification task are displayed in Table III, and the result achieved in the signature 
recognition task can be seen in Table IV. The result obtained from the name components recognition task is shown in Table $\mathrm{V}$, and the results received from the name component verification task are displayed in Table VI. Finally, the results of the word spotting task can be seen in Table VII.

TABLE III. EERS OF THE SIGNATURE VERIFICATION TASK

\begin{tabular}{|c|c|c|c|c|c|}
\hline Rank & Algorithm & RFS & SFS & SiFS & Avg \\
\hline 1 & SCUT-CNN & $\mathbf{0 . 0 0 1 9}$ & $\mathbf{0 . 0 7 1 0}$ & $\mathbf{0 . 0 0 9 0}$ & $\mathbf{0 . 0 2 7 3}$ \\
\hline 2 & LTP+oBIFs & 0.0109 & 0.1091 & 0.0712 & 0.0637 \\
\hline 3 & ERL & 0.0302 & 0.1780 & 0.0955 & 0.1012 \\
\hline 4 & oBIFs & 0.0444 & 0.1876 & 0.1010 & 0.1110 \\
\hline 5 & LTP & 0.0511 & 0.1901 & 0.1105 & 0.1172 \\
\hline 6 & MDRL & 0.0986 & 0.2000 & 0.1258 & 0.1415 \\
\hline 7 & SRL & 0.1108 & 0.2045 & 0.1459 & 0.1537 \\
\hline 8 & RL400 & 0.1308 & 0.2145 & 0.1599 & 0.1686 \\
\hline 9 & RL & 0.1308 & 0.2145 & 0.1599 & 0.1686 \\
\hline \multicolumn{7}{|c|}{ Benchmark[7] } & 0.0201 & 0.1108 & 0.0031 & 0.0447 \\
\hline
\end{tabular}

TABLE IV. RA OF THE SIGNATURE RECOGNITION TASK

\begin{tabular}{|c|c|c|}
\hline Rank & Algorithm & Recognition Accuracy \\
\hline $\mathbf{1}$ & SCUT-CNN & $\mathbf{0 . 9 9 9 8}$ \\
\hline 2 & ERL & 0.9978 \\
\hline 3 & MDRL & 0.9901 \\
\hline 4 & SRL & 0.9874 \\
\hline 5 & RL400 & 0.9831 \\
\hline
\end{tabular}

TABLE V. RA OF THE NAME COMPONENTS RECOGNITION TASK

\begin{tabular}{|c|c|c|c|c|c|}
\hline Rank & Algo & FN & LN & $\begin{array}{c}\text { FN+LN } \\
\text { image level }\end{array}$ & $\begin{array}{c}\text { FN+LN } \\
\text { score level }\end{array}$ \\
\hline $\mathbf{1}$ & SCUT- & $\mathbf{0 . 9 9 7 7}$ & $\mathbf{0 . 9 9 2 2}$ & $\mathbf{0 . 9 9 8 4}$ & $\mathbf{1 . 0 0 0 0}$ \\
\hline 2 & ERL & 0.9901 & 0.9891 & 0.9921 & 0.9946 \\
\hline 3 & MDRL & 0.9811 & 0.9822 & 0.9898 & 0.9901 \\
\hline 4 & SRL & 0.9799 & 0,9801 & 0.9845 & 0.9876 \\
\hline 5 & RL400 & 0.9781 & 0.9811 & 0.9852 & 0.9874 \\
\hline
\end{tabular}

For signature verification results, as can be seen in Table III, nine (9) algorithms were submitted, appreciable verification performance was achieved from most of the submitted algorithms. The average performance of the algorithms submitted by Team SUT was the best for the average random, skilled and simple forgery experiment compared to the other algorithms submitted from other competitors. The performance gap to the next ranking system was about $\sim 0.02$., $\sim 0.1$, and $\sim 0.08$ for random, skilled and simple forgery experiments, respectively.

For signature recognition results, from Table IV, it can be concluded that the appreciable recognition accuracy was achieved in most of the submitted systems. The performance of the SCUT-CNN algorithm was the best compared to the other algorithms submitted in the competition, performance gap to the next ranking system was about 0.002 .

For name component recognition results, Table $\mathrm{V}$ shows that the appreciable verification performance was achieved in most of the submissions. The algorithm based on CNN of Team SCUT was the best compared to the other algorithms submitted in the competition; this applies to all types of the name components. Another important observation was that the combination of the feature (FN and LN combined) was effective to produce a better result; this result also applied to all teams' algorithms.

For name component verification results, it can be seen from Table VI that the appreciable verification performance was achieved in most of the submitted systems. The performance of the submission from Team SCUT on the last name was the best with respect to the average random, skilled and simple forgery experiments, compared to the other algorithms submitted in the competition. The performance gap to the next ranking system was about $\sim 0.09$ for the best average of random and skilled forgery experiments.

For short answer work spotting result, only one participant has submitted their results which can be seen in Table VII. Although a good precision rate was achieved, the recall and F1 measures were rather poor. This applies to both printed and handwritten short answer words. The number of short answers retrieved was considerably good, that is 434 and 425 for printed and cursive handwritten words, respectively.

TABLE VII. EER RESULTS OF THE ANSWER VERIFICATION TASK

\begin{tabular}{|c|c|c|c|c|c|c|}
\hline Rank & Algo & $\begin{array}{c}\text { No. } \\
\text { Word }\end{array}$ & Type & Precision & Recall & F1 \\
\hline 1 & \multirow{2}{*}{ IF } & 434 & Printed & 0.90907 & 0.5376 & 0.6490 \\
\cline { 3 - 7 } & & 425 & Cursive & 0.90141 & 0.58036 & 0.6706 \\
\hline
\end{tabular}

For short answer recognition results, it can be concluded from Table VIII that only a small improvement of $0.07 \%$ was achieved by the best algorithm, ERL, when compared to the benchmark of [11]. Hence, it is essential to concentrate on investigating this problem in the future.

TABLE VIII. EER RESULTS OF THE ANSWER VERIFICATION TASK

\begin{tabular}{|c|c|c|}
\hline Rank & Algorithm & RA in \% \\
\hline 2 & ERL & 91.12 \\
\hline 3 & MDRL & 86.18 \\
\hline 4 & SRL & 82.08 \\
\hline 5 & RL400 & 81.30 \\
\hline & {$[11]$} & 91.05 \\
\hline
\end{tabular}

Way forward: In both the recognition and verification tasks, CNN based model had outperformed the traditional handcrafted features and have shown effective development in most of the experimental scenarios. For the recognition task, CNN model worked better than handcrafted feature by a great extent. For the signature and name component recognition tasks, good accuracy was achieved. However, the challenge is when a system with more extensive data captured in non-ideal condition population size and longitudinal study will be handled. For signature and name component verification tasks, a better algorithm to handle skilled along with random and simple forgery scenarios is yet to be found, hence, requires a further investigation.

Relatively less interest was found among the participants for the answer recognition and answer (word) spotting task. Moreover, the results were not very impressive. Hence it will be essential to continue research in this direction.

\section{CONCLUSIONS AND FUTURE SCOPE}

The competition on Thai short answer assessment SASIGCOM 2020 was organised with the primary goals to record performance of short answer word spotting, Thai student signature and Thai name components recognition and verification, and to report the recent advancements in this area. Moreover, it also aims to provide a common platform to evaluate Thai signature and name components recognition and verification algorithms using unique datasets. Subsequently, the showcasing of the competition in one of the most recognised gatherings in the handwriting research community 
i.e. ICFHR 2020 and promoting them via different electronic means of communications, have also increased the interest of researchers on this particular subject of research.

Furthermore, it has also recorded the recent development in the field of word spotting, offline ASV and name component/writer identification and verification.

Moreover, the conceived competition has satisfactorily fulfilled all of the above aims, and the gain in popularity and interest of the participants were noteworthy. The algorithms submitted by the participants demonstrated appreciable results on the proposed datasets. The authors hope that the critical analysis was undertaken, and the results of the different algorithms will also provide a way forward for further research. One very important aspect of this competition is the availability of datasets that are made public, which is enriched with a wider variety of practical scenarios.

\section{REFERENCES}

[1] A. Sharma and D. B. Jayagopi, "Automated grading of handwritten essays,” ICFHR, 2018, pp. 279-284.

[2] E. Shaikh, et al., "Automated grading for handwritten answer sheets using convolutional neural networks," ICTCS, 2019, pp. 1-6.

[3] H. Suwanwiwat et al., "A complete automatic short answer assessment system with student identification," ICDAR 2015, pp. 611-615.

[4] A. Das, et al., (2014, December). A new wrist vein biometric system., 2014 IEEE SSCI (pp. 68-75).

[5] H. Suwanwiwat et. al., "ICFHR 2018 competition on thai student signatures and name components recognition and verification (tsncrv2018)," ICFHR), 2018, pp. 500-505.

[6] T. Putte and J. Keuning, Biometrical Fingerprint Recognition: Don't get your Fingers Burned. Boston, MA: Springer US, 2000, pp.289-303.
[7] A. Das. Face recognition in reduced Eigen-plane. In Communications, Devices and Intelligent Systems (CODIS), 2012 International Conference on (pp. 620-623). IEEE.

[8] A. Das and R. Parekh, "2012 : Iris recognition using a scalar based template in eigen-space," Int. Journal of Computer Science and Telecommunication, vol. 3, pp. 74-49.

[9] A. Das et al., "Multi-script versus single-script scenarios in automatic off-line signature verification," IET Biometrics, vol. 5, no. 4, pp. 305-313, 2016

[10] A. Das, et al., Sclera recognition-a survey. In Pattern Recognition ACPR 2013, pp. 917-921).

[11] H. Suwanwiwat et. al., "An Investigation of Discrete Hidden Markov Models on Handwritten Short Answer Assessment System", ICPRAI 2018.

[12] A. Das, et al., 'Thai Automatic Signature Verification Employing Textural Features', IET Biometrics, 2018

[13] H. Suwanwiwat, et al., 'An Automatic Student Verification System Utilising Off-Line Thai Name Components', DICTA, 2017, pp. 1-6.

[14] S. Lai, et al., (2018). Learning Discriminative Feature Hierarchies for Off-Line Signature Verification. ICFHR 2018, pp. 175-180).

[15] Y. Zhu, et al., Point-to-Set Similarity Based Deep Metric Learning for Offline Signature Verification. ICFHR2020.

[16] B. Graham, (2014). Fractional Max-Pooling. ArXiv Preprint ArXiv:1412.6071.

[17] C, L Liu, et al., (2004). Handwritten digit recognition: investigation of normalization and feature extraction techniques. Pattern Recognition, 37(2), 265-279.

[18] W. Bouamra, et al.,"Towards the design of an off-line signature verifier based on a small number of genuine samples for training," Elsivers J of ESA (2018). Vol. 107, 182-195

[19] I Hadjadj, et al., Offline Signature Verification Using Textural Descriptors. In IbPRIA 2019.

[20] C. Djeddi, et al., "Text-independent writer recognition using multiscript handwritten texts," Pattern Recognition Letters, 2013.

TABLE VI. EER OF THE NAME COMPONENT VERFICATION TASK

\begin{tabular}{|c|c|c|c|c|c|c|c|c|c|c|c|c|c|}
\hline \multirow{2}{*}{ Rank } & \multirow{2}{*}{ Algo } & \multicolumn{3}{|c|}{ FN } & \multicolumn{3}{|c|}{ LN } & \multicolumn{3}{|c|}{ FN+LN image level } & \multicolumn{3}{|c|}{ FN+LN score level } \\
\hline & & RSF & SFS & Avg & RSF & SFS & Avg & RSF & SFS & Avg & RSF & SFS & Avg \\
\hline 1 & SCUT-CNN & 0.0052 & 0.0574 & 0.0313 & 0.0023 & 0.0451 & 0.0237 & 0.0016 & 0.0292 & 0.0154 & 0.0004 & 0.0233 & 0.0118 \\
\hline 2 & ERL & 0.0600 & 0.0610 & 0.0650 & 0.0561 & 0.0575 & 0.0568 & 0.0504 & 0.0467 & 0.0485 & 0.0499 & 0.0416 & 0.04575 \\
\hline 3 & MDRL & 0.0908 & 0.0918 & 0.0913 & 0.0823 & 0.0875 & 0.0849 & 0.0805 & 0.0867 & 0.0836 & 0.0779 & 0.0716 & 0.07475 \\
\hline 4 & SRL & 0.1161 & 0.1255 & 0.1208 & 0.1081 & 0.1021 & 0.1051 & 0.0910 & 0.0935 & 0.0922 & $0 . .0870$ & 0.0800 & 0.0835 \\
\hline 5 & RL400 & 0.1656 & 0.1717 & 0.1687 & 0.1471 & 0.1492 & 0.1482 & 0.1140 & 0.1258 & 0.1199 & 0.1169 & 0.0958 & 0.1063 \\
\hline & [9] & 0.0004 & 0.1191 & 0.0598 & 0.0002 & 0.1111 & 0.0556 & $\mathrm{RP}$ & $\mathrm{RP}$ & $\mathrm{RP}$ & $\mathrm{RP}$ & $\mathrm{RP}$ & $\mathrm{RP}$ \\
\hline
\end{tabular}

$\mathrm{RP}=$ RESULT NOT PRESENT IN THE PUBLICATION, 